\title{
Síndrome de Intestino Corto
}

\author{
Pablo Franquelo Morales a, Mubarak Alramadan Alramadan, \\ Beatriz Valero Serrano ${ }^{a}$, Reagens Achille ${ }^{a}$
}

\author{
a Servicio de Urgencias, Hospital \\ Virgen de la Luz, Cuenca, \\ España. \\ b Servicio de Endocrinología, \\ Hospital Virgen de la Luz, \\ Cuenca, España. \\ Correspondencia: Pablo \\ Franquelo Morales, Servicio \\ de Urgencias, Hospital Virgen \\ de la Luz, C/ Hermandad de \\ Donantes de Sangre $n^{\circ} 2$, \\ 16002 - Cuenca. \\ E-mail: pfranquelo@yahoo.es
}

Recibido el 23 de diciembre de 2010.

Aceptado para su publicación el 9 de marzo de 2011.

\section{RESUMEN}

El síndrome de intestino corto es un cuadro clínico caracterizado por una importante disminución de la superficie intestinal efectiva por una pérdida anatómica o funcional del intestino delgado. Aparece sobre todo tras resecciones intestinales extensas por isquemia mesentérica. Sus principales complicaciones son diarrea, deshidratación, pérdida de peso, desnutrición, déficit de electrolitos, vitaminas y oligoelementos. Un soporte nutricional adecuado e individualizado constituye la base del tratamiento. Presentamos el caso de un varón joven con estado de hipercoagulabilidad y síndrome de intestino corto que presentó un síndrome confusional por abandono en la dieta y tratamiento, expresándose como una encefalopatía de Wernicke.

Palabras Clave. Síndrome de Intestino Corto, Terapia Nutricional, Encefalopatía de Wernicke.

\section{ABSTRACT}

\section{Short bowel syndrome}

Short bowel syndrome is characterized by a significant reduction in the effective intestinal surface by an anatomical or functional loss of the small intestine. It mainly occurs after extensive bowel resection due to mesenteric ischemia. The main complications are diarrhoea, dehydration, weight loss, malnutrition, and electrolyte, vitamin and trace element deficiency. Treatment is based on appropriate, individualized nutritional support. We report the case of a young man with a hypercoagulable state and short bowel syndrome who presented with a confusional state due to non-compliance to diet and treatment, expressed as a Wernicke encephalopathy.

Key words. Short Bowel Syndrome, Nutritional Therapy, Wernicke Encephalopathy.

\section{INTRODUCCIÓN}

El síndrome de intestino corto (SIC) es un cuadro clínico caracterizado por una importante disminución de la superficie intestinal efectiva por la pérdida anatómica o funcional del intestino delgado (ID) que aparece sobre todo tras resecciones extensas por isquemia mesentérica ${ }^{1}$ (tabla 1). Esto provoca diarrea, deshidratación, pérdida de peso, desnutrición, y déficit de electrolitos, vitaminas y oligoelementos como complicaciones fundamentales ${ }^{1-3}$ (tabla 2). Los pacientes con SIC precisarán un soporte nutricional adecuado e individualizado, inicialmente con nutrición parenteral total, y algunos de ellos de por vida, mientras en su intestino se producen cambios adaptativos para aumentar la absorción de nutrientes. Los avances en la nutrición han mejorado el pronóstico de estos pacientes a lo que se suman diversas técnicas quirúrgicas, como el trasplante intestinal, aunque conlleva una alta morbimortalidad ${ }^{4-7}$.

Se presenta un caso de encefalopatía de Wernicke (EW) en un paciente con $\mathrm{SIC}$, como manifestación de un síndrome confusional (SC) precipitado por el abandono de su dieta y tratamiento. 


\section{OBSERVACIONES CLÍNICAS}

Presentamos el caso de un varón de 36 años con disartria, marcha inestable, parestesias en manos y confusión mental de una semana de evolución que fue traído a urgencias por un familiar. En sus antecedentes personales había requerido una resección intestinal masiva del ID por trombosis de la vena mesentérica superior con necrosis extensa hacía unos años, con consecuencia de hiperparatiroidismo secundario a malabsorción, y seguía un tratamiento con complejos vitamínicos y acenocumarol, como tratamiento preventivo por su condición de hipercoagulabilidad familiar que predispuso al episodio trombótico. No refería hábito enólico ni tabáquico y en los últimos meses presentaba un importante descuido higiénico y dietético con suspensión del tratamiento tras pérdida del empleo y abandono del hogar por su esposa. Inicialmente atribuimos el cuadro a un estado depresivo con episodios esporádicos de intoxicación etílica, aunque nos aseguraron que no había consumido alcohol. En vista de que resultaba complicado realizar la anamnesis y la exploración física del paciente por falta de colaboración, se solicitaron pruebas complementarias $\mathrm{y}$, ante la sospecha de padecer una EW secundaria a un déficit nutricional, se administró tiamina intramuscular.

La exploración física mostró constantes vitales dentro de la normalidad, con marcada delgadez y palidez cutánea, lengua pastosa sin fetor enólico e importante sequedad de piel. El habla era disártrica, con auscultación cardiopulmonar, abdomen y extremidades sin hallazgos patológicos. La exploración neurológica sin rigidez de nuca ni signos meníngeos y con funciones corticales superiores, lenguaje y pares craneales normales. No afectación de la motilidad ocular, ni nistagmo, con fondo de ojo y campimetría visual sin hallazgos de interés. Sistema motor con reflejos osteotendinosos $3 / 4$, reflejos cutáneo plantares flexores, fuerza, tono y tropismo normales, con sensibilidades superficial y profunda sin hallazgos significativos, así como marcha atáxica e inestable.

En la analítica se objetivó una hipoproteinemia de $6,3 \mathrm{~g} / \mathrm{dL}$, con $\mathrm{pH}$ de 7,22 , bicarbonato de 5,7 $\mathrm{mmol} / \mathrm{L}$, magnesio de $0,9 \mathrm{mEq} / \mathrm{L}$, albúmina de 3,8 $\mathrm{g} / \mathrm{dL}$ e importante déficit de vitaminas $\mathrm{A}, \mathrm{B}$ y $\mathrm{D}$.

Se trataba de un paciente con SIC, que de por sí predispone a un déficit de tiamina, sumado a una dieta deficiente y al abandono de su medicación habitual, lo que permitió el desarrollo de un síndrome confusional que desapareció completamente en pocos días tras la administración de complejos vitamínicos, hierro, calcio, sulfato de zinc, una dieta de $2.000 \mathrm{Kcal}$ sin residuos, evitando alimentos ricos en grasas saturadas, y reintroducción posterior de acenocumarol.

\section{COMENTARIOS}

La EW es un trastorno causado por la deficiencia de tiamina que se presenta principalmente en personas con alcoholismo crónico y malnutrición, pudiendo ser prevenible. La clínica consiste habitualmente en la triada clásica de ataxia, oftalmoplejia y confusión, no obstante para su diagnóstico se requieren al menos dos de los siguientes criterios: dieta deficiente, alteraciones oculomotoras, disfunción del cerebelo y confusión mental ${ }^{8}$. Probablemente nuestro paciente con SIC, que de por si predispone a una malabsorción, presentó una EW como expresión de un síndrome confusional agudo, infrecuente en este tipo de pacientes, que fue precipitado por el menoscabo en el cuidado de la dieta y de su tratamiento habitual.

EI SIC se produce cuando tiene lugar una pérdida mayor del $70-75 \%$ de la longitud total del ID, pudiéndose conservar o no el colon, aunque existe una gran variabilidad individual. La gravedad del SIC depende no sólo de la longitud de ID que se pierde, pues además influyen otros factores como el segmento de ID afectado (peor cuando se compromete el íleon que otros segmentos más proximales), la conservación o no del estómago, la válvula ileocecal y/o el colon (más desfavorable si se resecan), la presencia de enfermedad en el intestino residual, el tiempo transcurrido de adaptación del intestino tras la resección (normalmente dura más de un año) y la edad del paciente, de manera que cuanto más joven, mayor es la capacidad de adaptación del intestino remanente ${ }^{1}$.

Las necesidades nutricionales de estos pacientes varían según la región de intestino resecada, puesto que cada parte del intestino tiene unas funciones determinadas y existen áreas más eficientes que otras, no obstante como consecuencia de la reducción de la superficie absortiva se produce un déficit en la absorción de la mayoría de los nutrientes, que se perderán por las heces en forma de diarrea provocando desnutrición calórica y proteínica, además de las complicaciones mencionadas ${ }^{1,9,10}$. 
El tratamiento nutricional adecuado constituye el factor clave para la evolución de los pacientes con SIC, presentando una importante variabilidad, por lo que debe ser individualizado. Las distintas posibilidades comprenden desde el soporte nutricional enteral o parenteral durante toda la vida en los pacientes que presentan múltiples complicaciones, hasta una dieta oral adaptada que permite una buena calidad de vida. El trasplante intestinal se presenta como la técnica quirúrgica más desarrollada y se reserva para los pacientes que precisen mantenerse de forma crónica con nutrición parenteral y desarrollen complicaciones graves, aunque todavía presenta una morbimortalidad importante ${ }^{11}$.

Con este caso pretendemos poner en conocimiento del médico de atención primaria el SIC, el cual puede presentar complicaciones a largo plazo, como un síndrome confusional en nuestro caso, y también destacar la importancia de un seguimiento estrecho y consensuado con el endocrinólogo para prevenirlas.

- Enfermedad de Crohn

- Compromiso de vasos mesentéricos (trombosis, embolia, hipoperfusión por shock, vólvulo o hernia estrangulada)

- Resecciones repetidas en enfermedad de Crohn

- Resección amplia en enteritis actínica

- $\quad$ Bypass intestinal en tratamiento de la obesidad

- Esclerodermia

- $\quad$ Síndrome de pseudoobstrucción intestinal crónica

- Traumatismo abdominal

- $\quad$ Síndrome obstructivo por adherencias

- Neoplasia intestinal

- Enteritis actínica

- Esprue

- Anormalidades del desarrollo en el período intrauterino (atresia yeyunal o ileal, vólvulo intrauterino, aganglionosis, gastrosquisis)

- Formas adquiridas en el período neonatal (vólvulo, enterocolitis necrosante)

Tabla 1. Causas de SIC.

\author{
Diarrea con o sin esteatorrea \\ Deshidratación y pérdida de electrolitos \\ Déficit de oligoelementos \\ Pérdida de peso \\ Colelitiasis \\ Nefrolitiasis \\ Osteomalacia \\ Hígado graso \\ Síndrome confusional \\ Acidosis metabólica \\ Ulcus gástrico \\ Déficit de vitaminas \\ Hipoabsorción de macronutrientes \\ Hipersecreción gástrica \\ Anemia \\ Sobrecrecimiento bacteriano \\ Cirrosis \\ Hiperparatiroidismo
}

Tabla 2. Complicaciones del SIC. 


\section{BIBLIOGRAFÍA}

1. Rodríguez A, Cánovas G. Síndrome de intestino corto en adultos. Tratamiento nutricional. Endocrinol Nutr. 2004; 51:163-72.

2. Fernández Bañares F, Esteve Comas M. Malabsorción intestinal. En: Farreras-Rozman, editor. Medicina Interna.16 ${ }^{a}$ ed. Barcelona: Elsevier España S.L.; 2009. p.198-9.

3. Binder HJ. Trastornos de la absorción. En: Kasper DL, Fauci AS, Longo DL, Braunwald E, Hauser SL, Jameson $\mathrm{JL}$, editores. Harrison Principios de Medicina Interna.16 ${ }^{\mathrm{a}}$. ed. Chile: McGraw-Hill Interamericana; 2005. p.1953-4.

4. American Gastroentrology Association Medical Position Statement. Short bowel syndrome and intestinal transplantation. Gastroenterology. 2003; 124:1105-10.

5. Thompson JS. Management of the short bowel syndrome. Gastroenterol Clin North Am. 1994; 23:403-20.
6. Scolapio JS. Treatment of short-bowel syndrome. Curr Opin Clin Nutr Metab Care. 2001; 4:557-60.

7. Scolapio JS. Short bowel syndrome. JPEN. 2002; 26:S115 .

8. Munir A, Hussain SA, Sondhi D, Ameh J, Rosner F. Wernicke's encephalopathy in a non-alcoholic man: case report and brief review. Mt Sinai J Med. 2001; 68:216-8.

9. Sundaram A, Koutkia P, Apovian CM. Nutritional management of short bowel syndrome in adults. J Clin Gastroenterol. 2002; 34:207-20.

10. Fukuchi S, Bankhead R, Rolandelli R. Parenteral nutrition in short bowel syndrome. En: Rombeau JL, Rolandelli $\mathrm{R}$, editors. Clinical nutrition. Parenteral nutrition. 3rd ed. Philadelphia: Saunders; 2001. p. 282-303.

11. AGA. Tecnical Review on short bowel syndrome and intestinal transplantation. Gastroenterology. 2003;124:111134. 